\title{
Association of physical activity with lower type 2 diabetes incidence is weaker among individuals at high genetic risk
}

\author{
Yann C. Klimentidis • Zhao Chen • Amit Arora • \\ Chiu-Hsieh Hsu
}

Received: 21 May 2014 / Accepted: 29 August 2014 / Published online: 2 October 2014

(C) Springer-Verlag Berlin Heidelberg 2014

\begin{abstract}
Aims/hypothesis We examined whether or not the association of physical activity with type 2 diabetes incidence differs according to several types of genetic susceptibility.

Methods In a large prospective cohort with 821 incident cases of type 2 diabetes, we examined interactions of physical activity with: (1) each of 65 type 2 diabetes-associated single nucleotide polymorphisms (SNPs); (2) a genetic risk score (GRS) comprising all 65 SNPs; (3) two GRSs comprised of SNPs implicated in insulin resistance (IR) and insulin secretion; (4) GRSs for fasting insulin (FI) and fasting glucose.

Results We found a significant interaction of physical activity and the type 2 diabetes GRS ( $p_{\text {interaction }}=0.016$ ), suggesting a weaker protective effect of physical activity in those at high genetic risk. Based on the interactions observed with the IR GRS $\left(p_{\text {interaction }}=0.046\right)$ and the FI GRS $\left(p_{\text {interaction }}=0.042\right)$, it appears that the overall type 2 diabetes GRS interaction most likely occurs through genetic susceptibility to IR as opposed to insulin secretion. Furthermore, this interaction was more pronounced in women $\left(p_{\text {interaction }}=0.0025\right)$ than in men $\left(p_{\text {interaction }}=0.46\right)$. No single SNP stood out as displaying a strong interaction with physical activity.

Conclusions/interpretation We conclude that although physical activity appears to have an overall protective effect on type 2 diabetes, this putative effect is weakest among individuals with high genetic risk for type 2 diabetes and IR.
\end{abstract}

Electronic supplementary material The online version of this article (doi:10.1007/s00125-014-3380-z) contains peer-reviewed but unedited supplementary material, which is available to authorised users.

Y. C. Klimentidis $(\square) \cdot$ Z. Chen $\cdot$ A. Arora $\cdot$ C.-H. Hsu Mel and Enid Zuckerman College of Public Health,

Division of Epidemiology and Biostatistics, University of Arizona,

1295 N. Martin, Tucson, AZ 85724, USA

e-mail: yann@email.arizona.edu
Keywords Genetic risk · Insulin resistance · Interaction · Physical activity $\cdot$ Type 2 diabetes

$\begin{array}{ll}\text { Abbreviations } \\ \text { ARIC } & \text { Atherosclerosis Risk in Communities } \\ \text { dbGaP } & \text { Database of Genotypes and Phenotypes } \\ \text { FG } & \text { Fasting glucose } \\ \text { FI } & \text { Fasting insulin } \\ \text { GRS } & \text { Genetic risk score } \\ \text { GWAS } & \text { Genome-wide association studies } \\ \text { IFG } & \text { Impaired fasting glucose } \\ \text { IR } & \text { Insulin resistance } \\ \text { NFG } & \text { Normal fasting glucose } \\ \text { SNP } & \text { Single nucleotide polymorphism }\end{array}$

\section{Introduction}

It is well established that physical activity reduces type 2 diabetes risk [1]. However, the extent of protection afforded by physical activity may differ according to genetic factors. Several studies have examined the interaction of type 2 diabetes-associated genetic variants and physical activity with mixed results. Interactions of variants in $P P A R G, S L C 2 A 2$, $H N F 4 A$ and other candidate genes with physical activity have previously been identified (e.g. [2, 3]). More recently, in a prospective cohort of Swedish adults, Brito et al [4] tested the interaction of 17 single nucleotide polymorphisms (SNPs), identified through genome-wide association studies (GWAS) with physical activity, on incident type 2 diabetes, and found a significant interaction for a SNP in $H N F 1 B$.

Among the 65 SNPs identified through GWAS (e.g. [5]), some exert their effect on type 2 diabetes through a reduction in insulin sensitivity, while most appear to exert their effect through impaired beta cell function $[5,6]$. The most 
comprehensive and detailed examination of the phenotypic effects of type 2 diabetes-associated loci has recently delineated nine loci associated with beta cell function, and four loci with insulin resistance (IR) or insulin insensitivity [7].

In the present prospective cohort study, we examine the interaction of 65 type 2 diabetes-associated SNPs, collectively and individually, with baseline physical activity on incident type 2 diabetes. Then, to gain further insight into the genetic and physiological basis of the interactions, we consider a genetic risk score (GRS) based on SNPs implicated in beta cell function, a GRS based on SNPs implicated in insulin sensitivity, as well as GRSs for fasting insulin (FI) and fasting glucose (FG), hypothesising that the primary underlying mechanism through which physical activity is protective is through insulin sensitivity [8], for which FI is a proxy.

\section{Methods}

Sample We used data on 8,101 self-identified white participants in the Atherosclerosis Risk in Communities study (ARIC). The ARIC dataset was obtained from the Database of Genotypes and Phenotypes (dbGaP). Briefly, the ARIC study is a multicentre prospective study of atherosclerosis in men and women between 45 and 64 years of age [9]. We only included participants without type 2 diabetes at the first visit, as defined by an FG level $<6.9 \mathrm{mmol} / \mathrm{l}$, no report of taking type 2 diabetes medication or physician diagnosis. Participants were followed over a period of up to nearly 12 years (mean follow-up: 7.8 years). Incident type 2 diabetes cases were identified at one of three follow-up visits based on FG level and medication criteria. Given the age of incident type 2 diabetes in this study (between 45 and 73 years of age) it is unlikely that there are a significant number of type 1 diabetes cases. Time to incident type 2 diabetes was extrapolated based upon glucose values at the ascertaining visit and the previous visit, as previously described [10]. Approval for this study was obtained from the University of Arizona Institutional Review Board.

Physical activity and dietary intake Each individual's level of physical activity was assessed using the Baecke Physical Activity questionnaire [11]. We focused on the sport and exercise components of physical activity. The 'sports index' in ARIC takes into account the frequency, duration and assigned intensity of habitual sports/exercise activity in the past year, as well as the self-rated amount of each activity compared with other individuals of the same age. The resulting semi-continuous index ranges from 1 (lowest level of activity) to 5 (highest level of activity) in increments of 0.25 . Further details about the questionnaire can be found elsewhere [12]. Usual dietary intake of fibre and carbohydrates was estimated from a semi-quantitative food frequency questionnaire as described elsewhere [13].

Genetic markers and risk scores We selected 65 SNPs confidently associated with type 2 diabetes and their respective risk alleles and effect sizes as listed in Morris et al [5] (ESM Table 1). We also selected 36 and 17 SNPs associated with FG and FI, respectively, as identified by Scott et al [14] (ESM Tables 2 and 3). After standard quality-control procedures on genotypes (Affymetrix 6.0; Affymetrix, Santa Clara, CA, USA), we imputed genotypes with IMPUTE2 software (https://mathgen.stats.ox.ac.uk/impute/impute_v2.html\#home) and all individuals in the 1000 Genomes Project as reference data [15] in order to obtain genotypes for all the SNPs mentioned above. All SNPs had 'info' (imputation quality) scores of $>0.6$. GRSs were calculated as the weighted sum of risk alleles, with weights corresponding to the respective SNP effect size as estimated in the respective meta-analysis mentioned above. Based on the most comprehensive and recent assessment of the putative functional effects of type 2 diabetes loci [7], we created an unweighted GRS of IR based on the SNPs near or in the PPARG, KLF14, IRS1 and GCKR genes, and an unweighted GRS of beta cell function based on SNPs near or in the TCF7L2, SLC30A8, HHEX/IDE, CDKAL1, $C D K N 2 A / 2, T H A D A, D G K B, P R O X 1$ and $A D C Y 5$ genes. We chose to leave these GRSs unweighted as the SNPs were identified based on a collection of many different measures of both insulin secretion and insulin sensitivity [7], making it difficult to assign a single weight to the respective SNPs.

Statistical analysis Within each of the nine strata defined by the type 2 diabetes GRS and physical activity tertiles, we calculated incidence rates by dividing the number of incident cases by the corresponding person time (incidence per 10,000 person-years of follow-up). We fit Cox proportional hazards regression models, implemented in R [16], to estimate HRs for the interactions of physical activity with SNPs and GRSs on type 2 diabetes incidence, and for the association of physical activity with type 2 diabetes incidence in different GRS and physical activity strata. We also considered type 2 diabetes incidence among those with normal fasting glucose (NFG; $<5.6 \mathrm{mmol} / \mathrm{l}$ ) and impaired fasting glucose (IFG; $\geq 5.6 \mathrm{mmol} / 1$ and $<6.9 \mathrm{mmol} / \mathrm{l}$ ) at baseline, as well as the incidence of IFG among those with NFG at baseline. Sex, age, BMI, smoking, and dietary fibre and total carbohydrate intake were included as covariates in all analyses. Interactions were modelled by including the product of physical activity and each SNP or GRS. To explore GRS-by-physical activity interactions, we stratified the sample according to low, intermediate and high GRS tertiles, and examined associations of physical activity with type 2 diabetes in each group. Stratification was also performed according to physical activity level in order to examine the association of GRSs with type 2 diabetes 
incidence within each tertile of physical activity. We also examined interactions separately by sex.

In order to verify that the assumptions of Cox proportional hazards regression were met, we tested the proportionality of the hazards over time by including a time-dependent covariate consisting of the interaction of the logarithm of the time to event, physical activity and the GRS. There was not sufficient evidence to reject the null hypothesis of hazard proportionality over time in the physical activity-by-GRS interactions ( $p>0.05$ ), showing that the current model is appropriate. For the GRS-by-physical activity interaction tests, we considered statistical significance at $\alpha=0.05$. Although there are five such tests, they are not independent since there is overlap of SNPs and genomic regions in the GRSs. For the SNP-by-physical activity interaction tests, we considered a Bonferroni-adjusted $p$ value threshold for statistical significance $\left(\alpha=7.7 \times 10^{-4}\right)$.

\section{Results}

Baseline and incidence characteristics are shown in Table 1. Other than differences in the number of incident type 2 diabetes cases $(p<0.0001)$, we did not observe any significant differences in baseline characteristics across type 2 diabetes GRS tertiles. All GRSs were weakly correlated with each other except for the beta cell and type 2 diabetes GRSs $\left(r^{2}=\right.$ 0.3) (ESM Table 4). In the Cox proportional hazards regression model considering only main effects, we found that BMI $\left(\mathrm{HR}_{\text {main }}=1.13,95 \%\right.$ CI $\left.1.12,1.15 ; p<2 \times 10^{-16}\right)$, the type 2 diabetes GRS $\left(\mathrm{HR}_{\text {main }}=1.91,95 \%\right.$ CI 1.64, 2.22; $p<2 \times$ $\left.10^{-16}\right)$, smoking $\left(\mathrm{HR}_{\operatorname{main}}=1.0005,95 \%\right.$ CI $1.0003,1.001$; $\left.p=8.16 \times 10^{-10}\right)$ and dietary carbohydrate intake $\left(\mathrm{HR}_{\text {main }}=\right.$ $1.001,95 \%$ CI 1.000, 1.002; $p=0.040$ ) were positively associated with type 2 diabetes incidence. In the entire sample, although physical activity was not significantly associated with type 2 diabetes incidence, the $\mathrm{HR}$ was $<1\left(\mathrm{HR}_{\text {main }}=\right.$
$0.938,95 \%$ CI $0.857,1.028 ; p=0.17)$, suggesting an overall protective effect of physical activity on type 2 diabetes.

None of the 65 type 2 diabetes SNPs showed a significant interaction with physical activity, after correction for multiple testing. Four SNPs showed nominally significant interactions with physical activity (rs1496653 in UBE2E2, $p=0.0009$; rs6795735 in ADAMTS9, $p=0.014$; rs10842994 in KLHDC5, $p=0.016$; and rs2943640 in IRS1, $p=0.038$ [ESM Table 1]). The IRSI SNP is designated as an IR SNP [7].

Figure 1 shows the incidence rates in each of nine strata defined by type 2 diabetes GRS and physical activity tertile. The incidence among physically active individuals was lower than the incidence among physically inactive individuals across all three strata of type 2 diabetes GRS. However, the decrease in incidence among active vs inactive individuals was steepest among the low GRS individuals.

We found a significant interaction of the type 2 diabetes GRS with physical activity on type 2 diabetes incidence $\left(\mathrm{HR}_{\text {interaction }}=1.27,95 \%\right.$ CI 1.04, 1.54; $\left.p=0.016\right)$. As shown in Fig. 2, we found that the association of physical activity with type 2 diabetes incidence was strongest in the low GRS group $\left(\mathrm{HR}_{\text {main }}=0.82,95 \% \mathrm{CI} 0.67,1.00 ; p=0.053\right)$ and weakest in the high GRS group $\left(\mathrm{HR}_{\text {main }}=1.04,95 \% \mathrm{CI}\right.$ $0.91,1.19 ; p=0.56$ ). Conversely, the association of the type 2 diabetes GRS with type 2 diabetes incidence was strongest among the individuals with the highest level of physical activity (ESM Table 5). We found a significant interaction of the type 2 diabetes GRS with physical activity $\left(\mathrm{HR}_{\text {interaction }}=\right.$ $1.59,95 \%$ CI 1.17, 2.15; $p=0.0025$ ) in women, but not in men $\left(\mathrm{HR}_{\text {interaction }}=1.10,95 \%\right.$ CI $0.85,1.43 ; p=0.46$ [ESM Table 6]). As in the entire sample, the putative protective effect of physical activity on type 2 diabetes in women was stronger among those with a low type 2 diabetes GRS compared with those with a higher type 2 diabetes GRS (ESM Table 7).

We found a nominally significant interaction of the IR GRS and physical activity $\left(\mathrm{HR}_{\text {interaction }}=1.07,95 \% \mathrm{CI} 1.00,1.15\right.$; $p=0.046$ ), but no evidence of an interaction of the beta cell

Table 1 Baseline and incidence characteristics in the overall sample and in each tertile of type 2 diabetes GRS

\begin{tabular}{lllll}
\hline & Overall $(n=8,101)$ & Low type 2 diabetes GRS & Intermediate type 2 diabetes GRS & High type 2 diabetes GRS \\
\hline Age (years) & $54.2 \pm 5.7$ & $54.2 \pm 5.7$ & $54.2 \pm 5.6$ & $54.0 \pm 5.6$ \\
Women (\%) & 53.6 & 54.5 & 52.8 & 53.5 \\
BMI $\left(\mathrm{kg} / \mathrm{m}^{2}\right)$ & $26.7 \pm 4.6$ & $26.7 \pm 4.6$ & $26.7 \pm 4.6$ & $26.6 \pm 4.6$ \\
Smoking (cigarette years) & $331 \pm 428$ & $330 \pm 427$ & $336 \pm 438$ & $327 \pm 421$ \\
Dietary fibre intake (g/day) & $17.6 \pm 8.1$ & $17.5 \pm 8.1$ & $17.5 \pm 8.0$ & $17.7 \pm 8.1$ \\
Dietary carbohydrate intake (g/day) & $199.0 \pm 83.0$ & $198.1 \pm 81.2$ & $198.3 \pm 82.8$ & $200.7 \pm 84.8$ \\
Physical activity (sport) index & $2.6 \pm 0.8$ & $2.5 \pm 0.8$ & $2.6 \pm 0.8$ & $2.6 \pm 0.8$ \\
Incident cases (n) & 821 & 180 & 296 & 345 \\
\hline
\end{tabular}

Values are mean \pm SD unless indicated otherwise

Cigarette years: Average number of cigarettes smoked per day multiplied by the number of years smoked

${ }^{a}$ Indicates a significant difference among type 2 diabetes GRS tertiles 


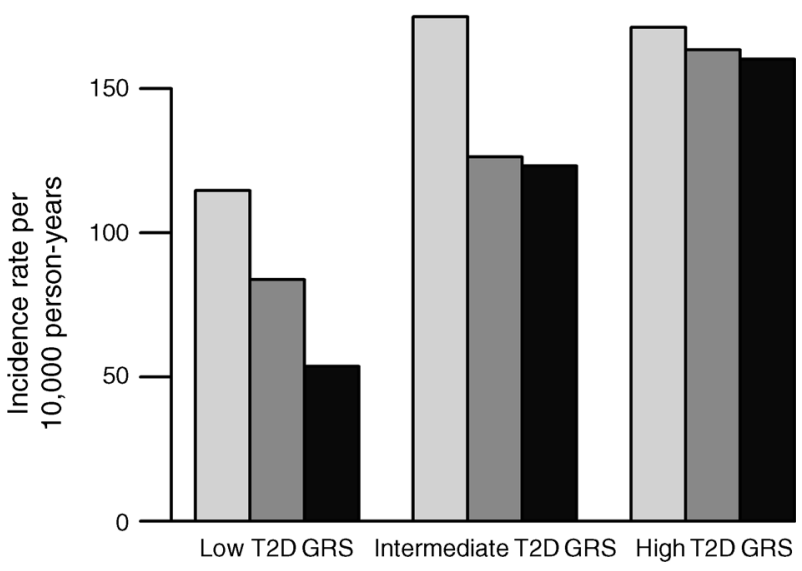

Fig. 1 Incidence rates of type 2 diabetes per 10,000 person-years across strata of type 2 diabetes (T2D) GRS and physical activity tertile. Light grey bar, low levels of physical activity; medium grey bar, intermediate levels of physical activity; black bar, high levels of physical activity

GRS with physical activity $\left(\mathrm{HR}_{\text {interaction }}=1.00,95 \%\right.$ CI 0.96 , $1.05 ; p=0.89$ ). We detected a significant interaction of the FI GRS with physical activity $\left(\mathrm{HR}_{\text {interaction }}=8.34,95 \%\right.$ CI 1.08 , $64.33 ; p=0.042$ ), but not of the FG GRS with physical activity $\left(\mathrm{HR}_{\text {interaction }}=1.10,95 \% \mathrm{CI} 0.46,2.62 ; p=0.83\right)$. As shown in Fig. 2, for both the IR and the FI interactions we observed the same trend as that observed for the type 2 diabetes GRS in that the association of physical activity with type 2 diabetes incidence was strongest in the people with low genetic risk. As with the type 2 diabetes GRS, we also observed that the association of these GRS with type 2 diabetes incidence was strongest among individuals with the highest level of physical activity (ESM Table 5). The interactions are not statistically significant in women or men for both the IR GRS

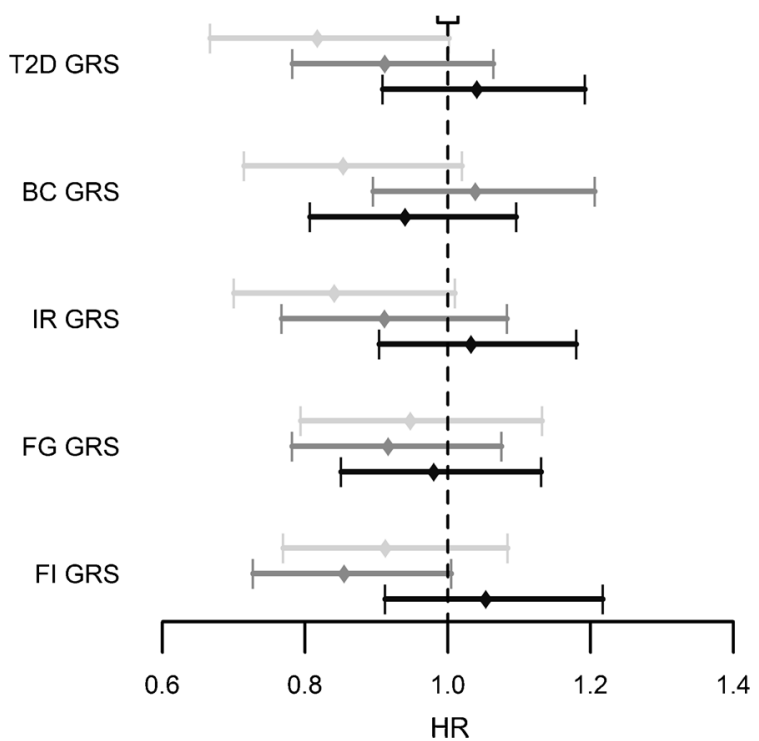

Fig. 2 HRs and 95\% CIs for type 2 diabetes per 1 unit increase in physical activity in three strata of type 2 diabetes (T2D), beta cell (BC), IR, FG and FI GRS. Physical activity is measured as an index between 1 and 5. Light grey lines, low GRS; medium grey lines, intermediate GRS; black lines, high GRS
$\left(\mathrm{HR}_{\text {interaction }}=1.11,95 \%\right.$ CI $0.99,1.24 ; p=0.075$ in women; $\mathrm{HR}_{\text {interaction }}=1.07,95 \% \mathrm{CI} 0.97,1.17 ; p=0.17$ in men), and for the FI GRS $\left(\mathrm{HR}_{\text {interaction }}=25.74,95 \%\right.$ CI $0.96,688.05$; $p=0.053$ in women, $\mathrm{HR}_{\text {interaction }}=4.31,95 \%$ CI $0.30,61.96$; $p=0.28$ in men [ESM Table 6]). We found that no single FI SNP*physical activity interaction reached statistical significance (ESM Table 3).

Finally, we found a similar, albeit weaker, pattern of interaction of the GRSs with physical activity on type 2 diabetes incidence among those with NFG or IFG at baseline (ESM Table 8), and on IFG incidence among those with NFG at baseline (ESM Table 9).

\section{Discussion}

In the ARIC prospective cohort study we have shown a statistically significant interaction of baseline physical activity and type 2 diabetes genetic risk on type 2 diabetes incidence. Specifically, we observed that the association between physical activity and type 2 diabetes incidence is weaker in those with higher type 2 diabetes genetic risk $\left(\mathrm{HR}_{\text {main }}=1.04 ; p=0.56\right)$ than those with lower type 2 diabetes genetic risk $\left(\mathrm{HR}_{\text {main }}=0.82\right.$; $p=0.053$ ). Reciprocally, the association of the type 2 diabetes GRS with type 2 diabetes incidence is strongest among those with a high level of physical activity. Furthermore, our results suggest that this interaction is principally driven through genetic risk to IR and is most pronounced in women.

The strengths of our study include the use of a prospective cohort design, which reduces the potential for reverse causation between physical activity and type 2 diabetes; the use of a relatively large panel of 65 SNPs to define genetic risk; and the genetic dissection of the pathophysiological process that may be implicated in this effect modification. In addition, we have controlled for several potential important confounders such as diet, smoking and BMI. The adjustment for BMI, in particular, suggests that the associations and interactions of physical activity and genetic risk on type 2 diabetes incidence are independent of obesity. The weaknesses of our study include the use of a subjective measure of physical activity based on questionnaire responses, and the fact that the GRS explains only a fraction of the expected heritability, hence the likely low statistical power to detect interactions with individual SNPs. Our finding of stronger interactions in women is likely not explained by statistical power differences since the sample sizes of men and women were roughly equal, and the number of incident cases was higher among men.

Although physical activity generally protects against type 2 diabetes, our findings suggest that the level of protection conferred by physical activity is lower in individuals (especially women) with a higher genetic risk for type 2 diabetes or IR. Previous studies have pointed to the effect of exercise on decreasing IR (e.g. [8]), indicating that the protective effect of 
physical activity on type 2 diabetes risk occurs principally through the mechanism of greater insulin sensitivity.

Our finding that the association of genetic risk with type 2 diabetes is strongest in those who are most physically active contrasts with the fairly well established interaction of physical activity and genetic risk for obesity, whereby the association of FTO or a BMI GRS with BMI is weakest in individuals who are physically active [17-19]. However, our results are similar to those recently obtained by Langenberg and colleagues [20] in which the putative effect of type 2 diabetes genetic risk was found to be strongest among younger, leaner and more physically active individuals (although the latter interaction with physical activity was not statistically significant in that study).

In conclusion, we have found an interaction of genetic risk with physical activity on incident type 2 diabetes that appears to be driven principally through genetic risk to IR. In future studies, it will be important to use less subjective measures of physical activity, to replicate this finding in another large prospective cohort study, to better understand the sex difference observed, and to generalise this finding to other age and ethnic/racial groups.

Acknowledgements Data from the ARIC study was obtained from dbGaP through accession numbers: phs000280.v2.p1. The ARIC study is carried out as a collaborative study supported by National Heart, Lung, and Blood Institute contracts (HHSN268201100005C, HHSN268201100006C, HHSN268201100007C, HHSN268201100008C, HHSN268201100009C, HHSN $268201100010 \mathrm{C}$, HHSN $268201100011 \mathrm{C}$, and HHSN268201100012C). Funding for GENEVA was provided by National Human Genome Research Institute grant U01HG004402

(E. Boerwinkle, University of Texas Health Science Center, Houston, TX, USA). The authors thank the staff and participants of the ARIC study for their important contributions. We also thank A. Chougule (University of Arizona, Tucson, AZ, USA) for help with genotype imputation.

Funding YCK was supported by NIH grant K01DK095032.

Duality of interest The authors declare that there is no duality of interest associated with this manuscript.

Author contributions YCK contributed to the conception, design, analysis and interpretation of data, and writing of the manuscript. AA contributed to the design and analysis of data, and to the critical revision of the manuscript for important intellectual content. $\mathrm{ZC}$ and $\mathrm{CH}$ contributed to the analysis and interpretation of data, and to the critical revision of the manuscript for important intellectual content. All authors gave final approval of the version to be published. YCK is responsible for the integrity of the work as a whole.

\section{References}

1. Sigal RJ, Kenny GP, Wasserman DH et al (2006) Physical activity/ exercise and type 2 diabetes: a consensus statement from the American Diabetes Association. Diabetes Care 29:1433-1438
2. Nelson TL, Fingerlin TE, Moss LK et al (2007) Association of the peroxisome proliferator-activated receptor gamma gene with type 2 diabetes mellitus varies by physical activity among non-Hispanic whites from Colorado. Metabolism 56:388-393

3. Kilpeläinen TO, Lakka TA, Laaksonen DE et al (2007) Physical activity modifies the effect of SNPs in the SLC2A2 (GLUT2) and ABCC8 (SUR1) genes on the risk of developing type 2 diabetes. Physiol Genomics 31:264-272

4. Brito EC, Lyssenko V, Renström F et al (2009) Previously associated type 2 diabetes variants may interact with physical activity to modify the risk of impaired glucose regulation and type 2 diabetes. Diabetes 58:1411-1418

5. Morris AP, Voight BF, Teslovich TM et al (2012) Large-scale association analysis provides insights into the genetic architecture and pathophysiology of type 2 diabetes. Nat Genet 44 : 981-990

6. Florez JC (2008) Newly identified loci highlight beta cell dysfunction as a key cause of type 2 diabetes: where are the insulin resistance genes? Diabetologia 51:1100-1110

7. Dimas AS, Lagou V, Barker A et al (2014) Impact of type 2 diabetes susceptibility variants on quantitative glycemic traits reveals mechanistic heterogeneity. Diabetes 63:2158-2171

8. Segal KR, Edano A, Abalos A et al (1991) Effect of exercise training on insulin sensitivity and glucose metabolism in lean, obese, and diabetic men. J Appl Physiol 71:2402-2411

9. The Aric Investigators (1989) The Atherosclerosis Risk in Communities (ARIC) Study: design and objectives. The ARIC investigators. Am J Epidemiol 129:687-702

10. Duncan BB, Schmidt MI, Pankow JS et al (2003) Low-grade systemic inflammation and the development of type 2 diabetes: the atherosclerosis risk in communities study. Diabetes 52:1799-1805

11. Baecke JA, Burema J, Frijters JE (1982) A short questionnaire for the measurement of habitual physical activity in epidemiological studies. Am J Clin Nutr 36:936-942

12. Folsom AR, Arnett DK, Hutchinson RG et al (1997) Physical activity and incidence of coronary heart disease in middle-aged women and men. Med Sci Sports Exerc 29:901-909

13. Diez-Roux AV, Nieto FJ, Caulfield L et al (1999) Neighbourhood differences in diet: the Atherosclerosis Risk in Communities (ARIC) Study. J Epidemiol Community Health 53:55-63

14. Scott R, Lagou V, Welch RP et al (2012) Large-scale association analyses identify new loci influencing glycemic traits and provide insight into the underlying biological pathways. Nat Genet 44:9911005

15. Howie B, Marchini J, Stephens M, Chakravarti A (2011) Genotype imputation with thousands of genomes. G3 (Bethesda) 1:457-470

16. Team RDC (2011) R: a language and environment for statistical computing.

17. Kilpeläinen TO, Qi L, Brage S et al (2011) Physical activity attenuates the influence of FTO variants on obesity risk: a metaanalysis of 218,166 adults and 19,268 children. PLoS Med 8: e1001116

18. Li S, Zhao JH, Luan J et al (2010) Physical activity attenuates the genetic predisposition to obesity in 20,000 men and women from EPIC-Norfolk prospective population study. PLoS Med 7: e1000332

19. Ahmad S, Rukh G, Varga TV et al (2013) Gene $\times$ physical activity interactions in obesity: combined analysis of 111,421 individuals of European ancestry. PLoS Genet 9:e1003607

20. Langenberg C, Sharp SJ, Franks PW et al (2014) Gene-lifestyle interaction and type 2 diabetes: the EPIC interact case-cohort study. PLoS Med 11:e1001647 